\title{
Метод адаптивного управления развитием инфокоммуникационных инфраструктур
}

\author{
Абдалов А.В., Гришаков В.Г., Логинов И.В. \\ Академия ФСО России, \\ Россия, 302015, г. Орел, ул. Приборостроительная, д. 35 \\ E-mail: senya@academ.msk.rsnet.ru,gvg@academ.msk.rsnet.ru, loginov_iv@bk.ru
}

\begin{abstract}
Аннотация. В работе рассмотрена проблема потокового управления обслуживанием заявок на создание и модернизацию инфокоммуникационных сервисов в крупномасштабных инфокоммуникационных инфраструктурах. Для инфраструктур с интенсивностью заявок на модернизацию более 10 заявок в сутки и несколькими источниками ресурсов предложена схема адаптивного управления обслуживанием заявок на создание/модернизацию инфокоммуникационных сервисов. Разработанная схема адаптивного управления представляет собой двухконтурную схему управления, позволяющую более эффективно обслуживать заявки на создание/модернизацию инфокоммуникационных сервисов. Первый контур управления обеспечивает распределение ресурсов по заявкам и адаптацию механизмов управления очередью. Второй контур управления управляет источниками и потоками ресурсов. Произведена экспериментальная оценка с использованием разработанной схемы адаптивного управления, которая показала её эффективность при практическом применении. Отмечен прирост целевого эффекта в условиях нестационарного потока ресурсов по сравнению с альтернативной схемой без использования схемы адаптивного управления.
\end{abstract}

Ключевые слова: ИТ-сервисы, распределение ресурсов, адаптивное управление, ИТинфраструктура.

Для цитирования: Абдалов А.В., Гришаков В.Г., Логинов И.В. 2021. Метод адаптивного управления развитием инфокоммуникационных инфраструктур. DOI. Экономика. Информатика, 48 (4): 784-793. DOI: $10.52575 / 2687-0932-2021-48-4-784-793$.

\section{The method of adaptive management of the development of information and communication infrastructures}

\author{
Arsentii V. Abdalov, Vadim G. Grishakov, Ilya V. Loginov \\ Academy of the FSG of Russia, \\ 35 Priborostroitelnaya str., Orel, 302015, Russia \\ E-mail: senya@academ.msk.rsnet.ru,gvg@academ.msk.rsnet.ru, loginov_iv@bk.ru
}

\begin{abstract}
The paper considers the problem of stream management of applications for the creation and modernization of infocommunication services in large-scale infocommunication infrastructures. For infrastructures with an intensity of requests for modernization of more than 10 requests per day and several sources of resources, a scheme for adaptive management of requests for the creation/modernization of information and communication services is proposed. The developed adaptive control scheme is a twocontour control scheme that allows more efficient servicing of applications for the creation/modernization of information and communication services. The first control loop ensures the distribution of resources according to requests and the adaptation of queue management mechanisms. The second control loop manages the sources and flows of resources. An experimental assessment was made using the developed adaptive control scheme, which showed its effectiveness in practical application. An increase in the target effect in the conditions of a non-stationary flow of resources is noted in comparison with the alternative scheme without the use of an adaptive control scheme.
\end{abstract}

Keywords: service discipline, non-stationary resource flow, infocommunication services, resources, efficiency assessment, application. 
For citation: Abdalov A.V., Grishakov V. G., Loginov I. V. 2021. The method of adaptive management of the development of information and communication infrastructures. Economics. Information technologies, 48 (4): 784-793. (in Russian). DOI: 10.52575/2687-0932-2021-48-4-784-793.

\section{Введение}

Для современных корпораций характерно наличие крупномасштабных инфокоммуникационных (ИТ) инфраструктур. Изменение условий функционирования корпорации, конкурентная борьба, новые рынки, организационные изменения и вывод на рынок новых продуктов требуют постоянного создания новых автоматизирующих и информационных сервисов. Таким образом, в рамках развития ИТ-инфраструктуры организации непрерывно создаются и модернизируются ИТ-сервисы с использованием необходимых для них ресурсов. Управление развитием ИТ-инфраструктуры реализуется в рамках процессов проектирования и преобразования новых и изменяемых услуг (ГОСТ 20000-1-2013) или процесса управления каталогом услуг (ГОСТ 57193-2016). Сущность этих процессов определяется потребностью в автоматизации и информатизации деятельности корпорации, а также ограничениями по ресурсам на развитие ИТ-инфраструктуры. При стационарных потоках поступления ресурсов задача планирования развития решается известным математическим аппаратом.

Изменение же принципов и механизмов поступления ресурсов (это и прямые закупки, фонды, целевое и программное финансирование, спонсирование, поддержка государства, субсидирование) приводит к тому, что ресурсы для новых и модернизируемых ИТ-сервисов поступают в ИТ-подразделение из различных источников, отличающихся своими характеристиками по предоставлению ресурсов (время, интенсивность, задержка, вероятность). Существующие механизмы управления обслуживанием заявок в рамках процесса непрерывного улучшения ориентированы на прогнозируемый поток ресурсов и в условиях неопределенности нестационарных ситуаций приводят к резкому снижению показателей создания ИТ-сервисов, включая значительные сроки задержки в создании новых средств автоматизации, а также отказе от развертывания прорывных сервисов из-за проблем нехватки ресурсов. Для рассматриваемых условий, отличающихся как неопределенностью поступлений ресурсов, так и нестационарностью потока заявок на создание / модернизацию ИТ-сервисов известный аппарат теорий управления запасами, управления поставками не дает необходимого решения. Это обосновывает актуальность задачи разработки механизмов управления потоками ресурсов и обслуживанием заявок на модернизацию ИТ-инфраструктуры в условиях неопределенности характеристик потоков ресурсов.

\section{Анализ известных подходов к управлению развитием ИТ-инфраструктур при множественных источниках ресурсов}

В рамках решения задачи управления развитием ИТ-инфраструктуры организации [Бон, Кеммерлинг, Пондаман, 2003] управление распределением ресурсов для создания новых и модернизации существующих ИТ-сервисов опирается на инструментарий сложных комбинаторных задач управления. Практические рекомендации по распределению ресурсов для создания и модернизации ИТ-сервисов описаны в еTOM, ITIL. Приведенные в них модели характеризуются низкой формализацией процесса распределения ресурсов. Большая часть известных методов распределения ресурсов опирается на динамическое распределение ресурсов [Беллман, Дрейфус, 1965; Могилевский, 2005]. Другим подходом является метод распределения ресурсов на сетях - путем решения задач дискретной оптимизации, позволяющих минимизировать время распределения ресурса или упущенную выгоду в конкретной ситуации. Задачи распределения ресурсов существуют в законченном виде лишь для ограниченного ряда постановок [Бурков, Новиков, 2004; Воронин, Губко, Мишин, Новиков, 2005], основные же результаты в настоящее время лежат в плоскости прикладных 
исследований, направленных на разработку эффективных вычислительных методов и специализированных инструментальных средств, в том числе в условиях неопределенности исходных данных - потоков заявок и ресурсов.

Рассмотрим основные подходы к распределению лимитированных ресурсов при создании ИТ-сервисов. В [Воронин, Губко, Мишин, Новиков, 2005] формулируется и решается типовая задача распределения ресурсов между проектами, входящими в портфель ИТ-проектов. В [Карымов, Славская, 2001] рассматриваются новые методические подходы к решению статической задачи оптимального распределения ресурсов. Задачу статического распределения ресурсов предлагается решать в постановке теории покрытий. Задача динамического распределения ресурсов в условиях конфликта ставится и решается как дифференциальная игра.

Вторая группа методов решения задачи распределения ресурсов базируется на учете ценности заявок на ИТ-сервисы с точки зрения приоритетов. Например, в [Воронин, Скатков, 2014] предложены новые алгоритмы решения задачи оптимального распределения ресурса, использующие множества Парето и двусторонние прогностические оценки оптимума, получаемые по методу ветвей и границ. В [Матвеев, 2005] представлены результаты по разработке оптимизационной математической модели распределения ресурсов и проведено численное моделирование. В [Грехов, Ивенин, Кудрявцев, 2008] описана математическая модель и метод динамического программирования распределения ограниченных ресурсов.

Третья группа методов непосредственно предлагает учитывать ценность нового ИТ-сервиса. В [Горбанева, 2010] описана и исследована задача распределения ресурсов в иерархических системах управления качеством речной воды путем нахождения равновесия по Штакельбергу. В [Прилуцкий, Власов, 2005] строится общая математическая модель распределения ресурсов и упорядочения работ в многостадийных системах. В рамках построенной модели формализуется широкий класс задач планирования и управления. Здесь также известны работы зарубежных ученых, которые предлагают также учитывать ценность ИТ-сервисов [Lane, 2009; Weber, Current, Benton, 1991; Wei, Chien, Wang, 2005; Wynne, Schaeffer, 2005; Ernstoff, Vincenzini, 1999].

Одновременное поступление потоков ресурсов и заявок на ИТ-сервисы обуславливает необходимость совместного управления распределением ресурсов с управления источниками (путем формирования оптимальных планов заявок на ресурсы). Решение такой задачи требует дополнения аппарата распределения ресурсов, описанного выше механизмами управления источниками ресурсов и генерируемыми ими потоками ресурсов.

\section{Проблема управления развитием инфокоммуникационных инфраструктур}

В работе рассматривается проблема управления развитием инфокоммуникационных инфраструктур в условиях ограниченности ресурсов [Могилевский, 2005]. Для инфраструктур с интенсивностью заявок на модернизацию более 10 заявок в сутки и несколькими источниками ресурсов предложена схема адаптивного управления обслуживанием заявок на создание/модернизацию инфокоммуникационных сервисов. Формальная постановка задачи имеет следующий вид:

1) В ИТ-подразделение организации поступают заявки $\mathrm{K}=\left\{\mathrm{K}_{1}, \mathrm{~K}_{2}, \ldots, \mathrm{K}_{\mathrm{i}}\right\}$ на создание информационных сервисов с интенсивностью $\lambda^{K}$ в форме нестационарного потока.

2) Заявка представляет собой задачу по созданию или модернизации ИТ-сервиса в

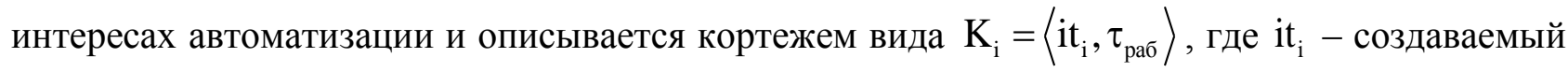
или модернизируемый ИТ-сервис, $\tau_{\text {раб }}-$ временной интервал в течение которого должен функционировать ИТ-сервис.

3) Создаваемый / модернизируемый ИТ-сервис $i t_{i}$ требует для своего создания определенного количества ресурсов различных видов и описывается кортежем 
$\mathrm{it}_{\mathrm{i}}=\left\langle\right.$ uid, Туре $\left., \mathrm{R}_{\mathrm{i}}, \tau_{\text {раб }}\right\rangle$, где $\mathrm{R}_{\mathrm{i}}(\mathrm{t})=\left(\mathrm{r}_{\mathrm{i} 1}, \mathrm{r}_{\mathrm{i} 2}, \ldots, \mathrm{r}_{\mathrm{im}}\right)$ вектор ИТ-ресурсов, $\mathrm{r}_{\mathrm{im}}-$ количество ресурсов Т-го вида, используемого в і-ом ИТ-сервисе, Туре - тип ИТ-сервиса, $\tau_{\text {раб }}-$ временной интервал в течение которого должен функционировать ИТ-сервис.

4) Ресурсы поступают из внешних источников $\mathrm{Q}_{\mathrm{i}}(\mathrm{t})=\left(\mathrm{Q}_{1}, \mathrm{Q}_{2}, \ldots, \mathrm{Q}_{\mathrm{n}}\right)$. Характеристики источников ресурсов различны, потоки ресурсов - нестационарны.

5) ИТ-подразделение генерирует заявки на ресурсы к каждому источнику и распределяет доступные ресурсы между заявками на ИТ-сервисы.

6) Выбор заявки на ИТ-сервис для реализации осуществляется в соответствии с внутренними правилами и критериями организации. При этом учитывается важность, категория сервиса, источник заявки, время развёртывания, ресурсоемкость.

\section{Схема адаптивного управления развитием инфокоммуникационных инфраструктур на основе совместного управления потоками ресурсов и обслуживанием заявок на создание/модернизацию ИТ-сервисов}

Для решения проблемы управления развитием инфокоммуникационных инфраструктур предложена схема адаптивного совместного управления обслуживанием заявок на создание/модернизацию ИТ-сервисов в крупномасштабных ИТ-инфраструктурах и потоками ресурсов для них (рисунок 1). Предложенный вариант представляет собой адаптивную систему, состоящую из устройства управления распределением ресурсов $Z^{\text {main }}(\mathrm{t})$ и двух контуров обратной связи - управления очередями $\mathrm{Z}^{\text {quеu }}(\mathrm{t})$ и управления источниками ресурсов $Z^{\text {source }}(\mathrm{t})$. Стоимостная функция $\mathrm{E}(\mathrm{t})$ максимизируется при выборе заявок для реализации (путем выделения ресурсов), учитывает важность ИТ-сервиса для автоматизации и задержку в создании ИТ-сервиса.

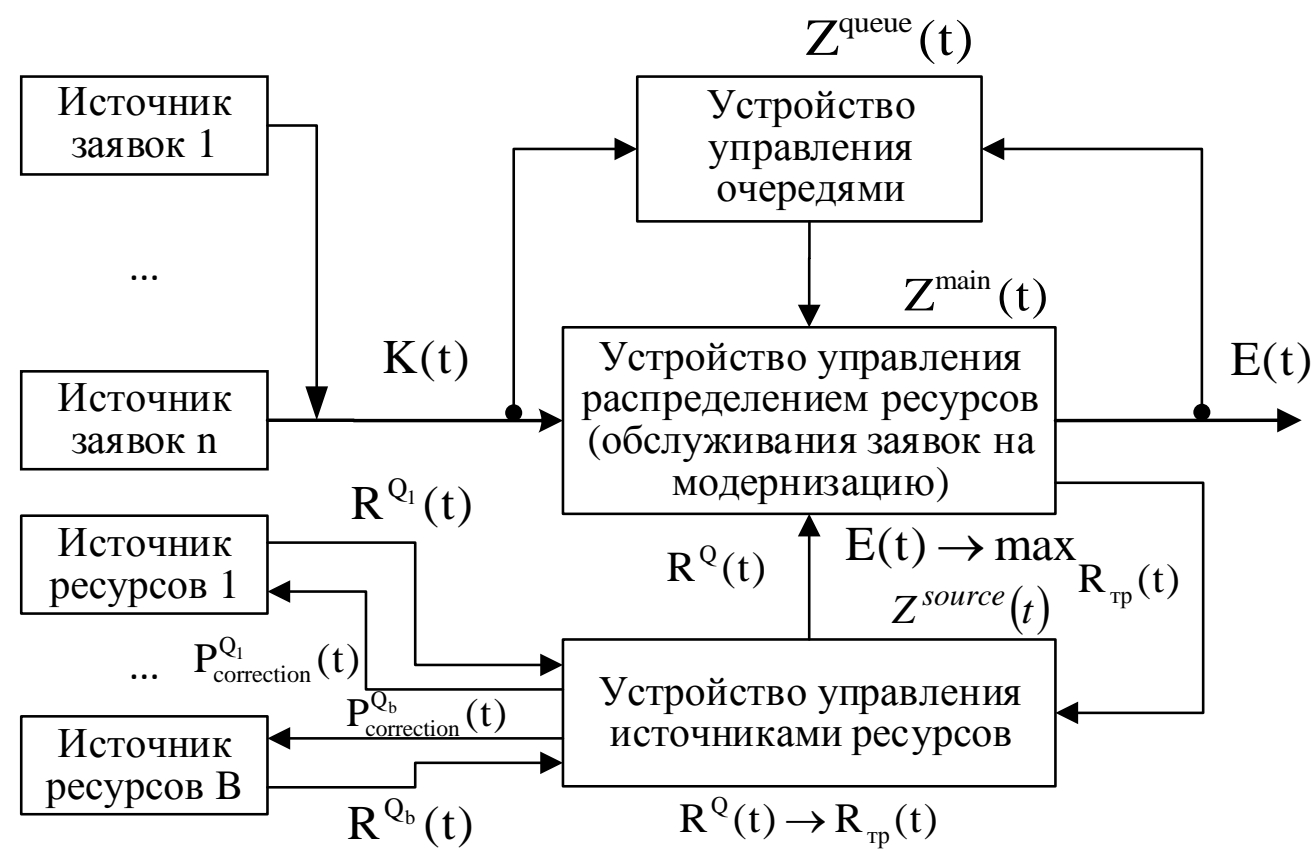

Рис. 1. Адаптивная система управления развитием ИТ-инфраструктуры

Fig. 1. Adaptive Management System for IT-infrastructure development

В систему поступают заявки $\mathrm{K}(\mathrm{t})$ на создание или модернизацию информационных сервисов. Поступившие заявки обрабатываются на устройстве управления распределением ресурсов $Z^{\text {main }}(\mathrm{t})$, которое обеспечивает распределение ресурсов по заявкам на ИТ-сервисы в соответствии с правилами обслуживания заявок $\mathrm{Z}^{\text {queu }}(\mathrm{t})$ и устройстве управления очередями - 
это является первым контуром управления. Задача устройства управления распределением ресурсов - наиболее оптимальным образом распределить ресурсы по ИТ-сервисам и получить наибольший эффект от их распределения $\mathrm{E}(\mathrm{t})$. Цель адаптивного управления устройством управления распределением ресурсов заключается в максимизации функционала:

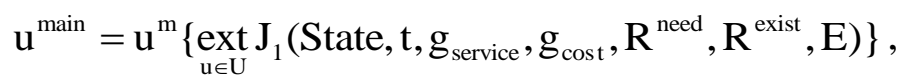

где $\mathrm{u}^{\mathrm{m}}$ - управляющие воздействия, State - состояние, в котором будет находится система, $\mathrm{t}$ - время, на котором применяется управляющее воздействие, $\mathrm{g}_{\text {service }}$ - функция распределения ресурсов по ИТ-сервисам, $\mathrm{g}_{\text {cost }}$ - функция оценки эффекта от распределения ресурсов по ИТ-сервисам, $\mathrm{R}^{\text {need }}$ - требуемые ресурсы, $\mathrm{R}^{\text {exist }}$ - ресурсы в наличии, Е - эффект, полученный на данном шаге.

Устройство управления очередями контролирует нахождение ИТ-сервисов в очереди и его задачей также является получение наибольшего эффекта $\mathrm{E}(\mathrm{t})$ для организации. Цель адаптивного управления устройства управления очередями:

$$
\mathrm{u}^{\text {queue }}=\mathrm{u}^{\mathrm{q}}\left\{\operatorname{ext}_{\mathrm{u} \in \mathrm{U}} \mathrm{J}_{1}\left(\text { State }, \mathrm{t}, \mathrm{g}_{\text {queue }}, \mathrm{E}\right)\right\},
$$

где $\mathrm{u}^{\mathrm{q}}$ - управляющие воздействия, State - состояние, в котором будет находится система, $\mathrm{t}$ - время, на котором применяется управляющее воздействие, $\mathrm{g}_{\text {queue }}-$ функция входных воздействий на очередь ИТ-сервисов, Е - эффект, полученный на данном шаге.

Управление источниками и потоками ресурсов осуществляется в устройстве управления источниками ресурсов - это второй контур адаптивного управления. Задачей данного устройства является приближение количества поступающих ресурсов к требуемым: $\mathrm{R}^{\text {exist }}(\mathrm{t}) \rightarrow \mathrm{R}^{\text {need }}(\mathrm{t})$. Данная проблема решается созданием плана корректировки для всех источников, существующих в организации. Цель адаптивного управления устройством управления ресурсами:

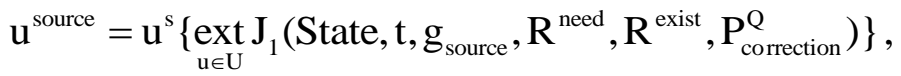

где $\mathrm{u}^{\mathrm{s}}$ - управляющие воздействия, State - состояние, в котором будет находиться система, $\mathrm{t}$ - время, на котором применяется управляющее воздействие, $\mathrm{g}_{\text {source }}-$ функция входных воздействий на источники поступления ресурсов, $\mathrm{R}^{\text {ned }}-$ требуемые ресурсы, $\mathrm{R}^{\text {exist }}$ - ресурсы в наличии, $\mathrm{P}_{\text {correction }}^{\mathrm{Q}}-$ план коррекции источников поступления ресурсов. Критерий оптимизации на данном шаге $\mathrm{R}^{\text {exist }} / \mathrm{R}^{\text {need }} \rightarrow 1$.

Рассмотрим работу предложенной адаптивной системы при обслуживании потока заявок на ИТ-сервисы. Система состоит из заявок на ИТ-сервисы $\mathrm{K}=\left\{\mathrm{K}_{1}, \mathrm{~K}_{2}, \ldots, \mathrm{K}_{\mathrm{i}}\right\}$ и peсурсов, имеющихся в наличии $\mathrm{R}^{\text {exist }}(\mathrm{t})=\left\{\mathrm{R}_{0}^{\text {exist }}(\mathrm{t}), \mathrm{R}_{1}^{\text {exist }}(\mathrm{t}), \ldots, \mathrm{R}_{\mathrm{m}}^{\text {exist }}(\mathrm{t})\right\}$. Для выполнения всех заявок $\mathrm{K}(\mathrm{t})$ необходимы ресурсы $\mathrm{R}^{\text {need }}(\mathrm{t})=\left\{\mathrm{R}_{0}^{\text {need }}(\mathrm{t}), \mathrm{R}_{1}^{\text {need }}(\mathrm{t}), \ldots, \mathrm{R}_{\mathrm{m}}^{\text {need }}(\mathrm{t})\right\}$. Количество ресурсов $\mathrm{R}^{\text {exist }}(\mathrm{t})<\mathrm{R}^{\text {need }}(\mathrm{t})$. У каждой заявки может быть 3 состояния - выполнена, отброшена, находится в очереди и обозначается через $\mathrm{S}_{\mathrm{j}}$. Состояние системы на каждом шаге $\mathrm{i}$ описывается следующим выражением:

$$
\text { State }_{\mathrm{i}}(\mathrm{t})=\left\{\sum_{\mathrm{j}=1}^{\mathrm{N}} \mathrm{S}_{\mathrm{j}}(\mathrm{t}), \mathrm{R}^{\text {exist }}(\mathrm{t})\right\} .
$$

Состояние, при котором выполняются все заявки, обозначим следующим выражением:

$$
\text { State }_{\max }(\mathrm{t})=\left\{\sum_{\mathrm{j}=1}^{\mathrm{N}} \mathrm{S}_{\mathrm{j}}(\mathrm{t}), \mathrm{R}^{\text {need }}(\mathrm{t})\right\}
$$


$\mathrm{B}$ состояние State $_{\max }(\mathrm{t})$ для организации получается максимальный эффект от выполнения заявок $\mathrm{E}_{\text {max }}(\mathrm{t})$. Соответственно эффект для i-го состояния обозначим через $\mathrm{E}_{\mathrm{i}}(\mathrm{t})$. Из-за того, что $\mathrm{R}^{\text {exist }}(\mathrm{t})<\mathrm{R}^{\text {need }}(\mathrm{t})$, то, следовательно, и $\mathrm{E}_{\mathrm{i}}(\mathrm{t})<\mathrm{E}_{\text {max }}(\mathrm{t})$. Соответственно первый контур управления позволяет управлять очередью заявок на ИТ-сервисы, в соответствии с потоком заявок и количеством поступающих ресурсов корректирует размер очереди, второй же контур управления помогает получить необходимое количество ресурсов в соответствии с текущим потоком заявок и потоком ресурсов от источников.

Исходя из выражений (1-5) цель адаптивного управления для всей системы в целом:

$$
\mathrm{u}^{\text {common }}=\mathrm{u}^{\mathrm{c}}\left\{\underset{\mathrm{u} \in \mathrm{U}}{\operatorname{ext}} \mathrm{J}_{1}\left(\text { State }, \mathrm{R}^{\text {exist }}, \mathrm{R}^{\text {need }}, \mathrm{t}, \mathrm{g}, \mathrm{E}\right)\right\},
$$

где $\mathrm{u}^{\mathrm{c}}$ - управляющие воздействия, State - состояние, в котором будет находиться система, $\mathrm{R}^{\text {exist }}$ - множество имеющихся ресурсов, $\mathrm{R}^{\text {need }}$ - множество необходимых ресурсов, $\mathrm{t}$ - время, на котором применяется управляющее воздействие, g - функция входных воздействий на систему с точки зрения распределения ресурсов, Е - эффект, полученный на данном шаге.

На основе разработанной схемы адаптивного управления выполнением заявок на ИТ-сервисы были реализованы алгоритмы, реализующие её функционал. Разработана программа, реализующая представленный механизм адаптивного управления и получено свидетельство о государственной регистрации программы для ЭВМ [Прилуцкий, Власов, 2005].

Данная схема будет позволять в условиях неопределенности поступления потоков ресурсов от источников управлять ими с учетом изменения характеристик потока заявок на ИТ-сервисы и обрабатывать поток поступающих заявок на ИТ-сервисы с учетом максимизации целевой функции, учитывающей совокупные эффекты автоматизации.

\section{Экспериментальная оценка эффекта адаптивного управления потоками ресурсов и обслуживанием заявок и обсуждение результата}

Для оценки эффекта предложенного варианта системы адаптивного управления были проведены экспериментальные исследования на базе разработанного стенда. В качестве входных данных для экспериментов были смоделированы нестационарные потоки поступающих ресурсов $\mathrm{N}^{\mathrm{R}}$, поступающих от 2 источников (рисунок 2, а), и потоки заявок на ИТ-сервисы $\mathrm{N}^{\mathrm{IT}}$ (рисунок 2, б). В ходе эксперимента смоделирован год работы ИТподразделения крупной организации. Типовой ситуацией при развитии ИТ-инфраструктур является вывод, что ресурсов, поступающих в организацию из внешних источников, будет недостаточно для выполнения всех входящих запросов. Смоделирована перегруженная ситуация, при которой отношение $\mathrm{K}$ требуемых ресурсов к доступным - варьировало в диапазоне 1,5-2,5 (рисунок 3 ).

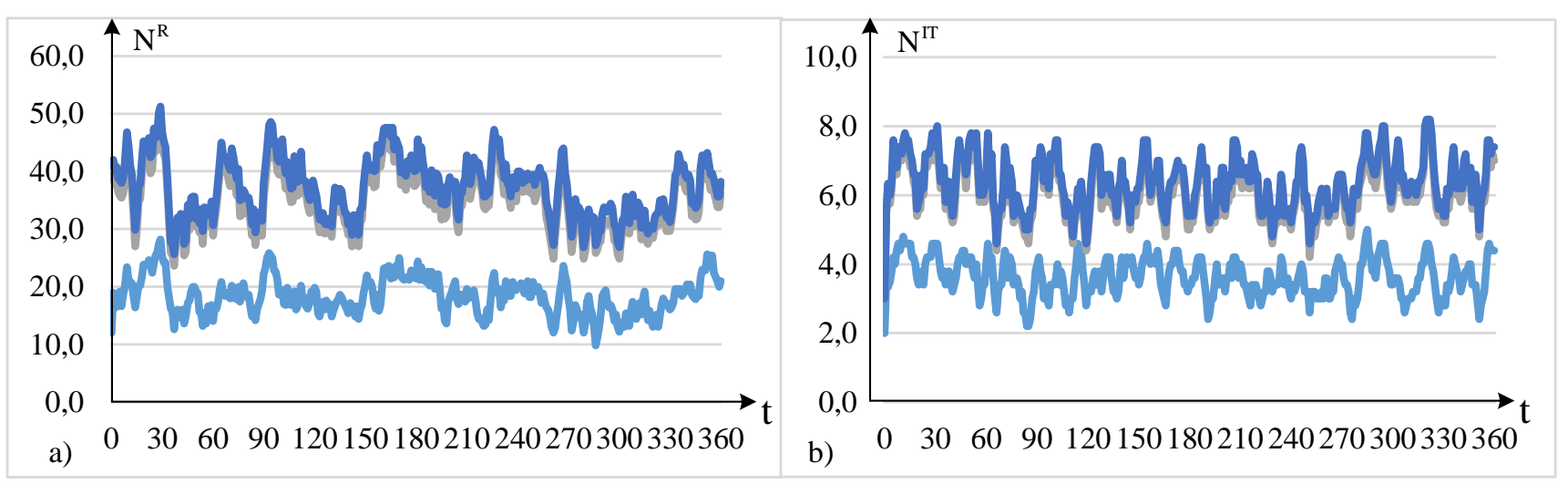

Рис. 2. Поток ресурсов и поток запросов на ИТ-услуги: а - нестационарный поток ресурсов (суммарный поток); б - стационарный поток запросов на ИТ-услуги (суммарный поток)

Fig. 2. The flow of resources and the flow of requests for IT services: a - non-stationary flow of resources (total flow); b - stationary flow of requests for IT services (total flow) 


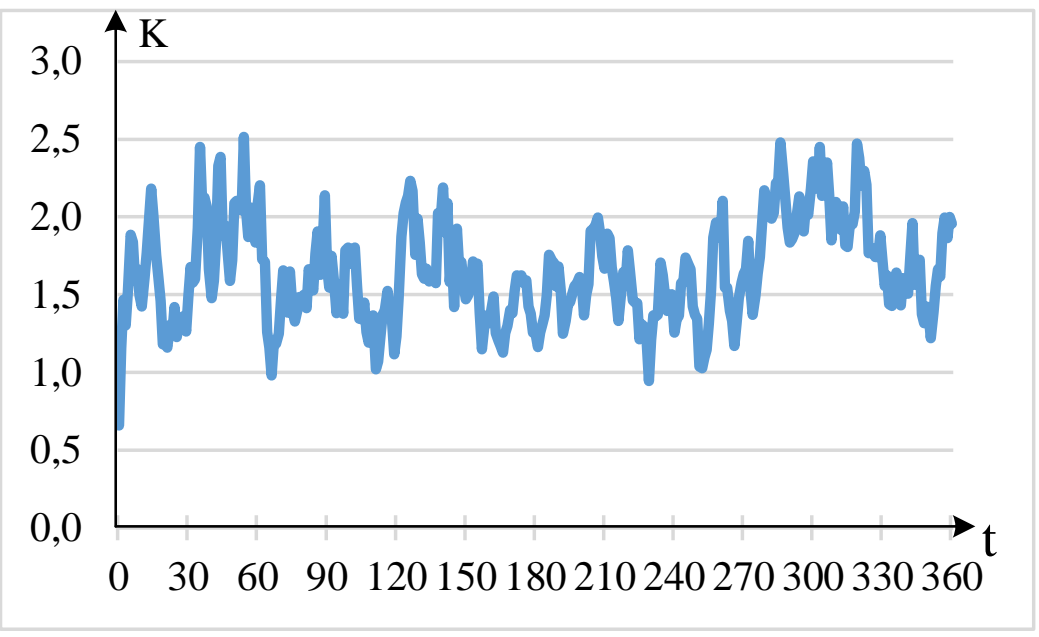

Рис. 3. Соотношение требуемых ресурсов к ресурсам, которые есть в наличии

Fig. 3 . The ratio of the required resources to the resources that are available

Сравнительный анализ нового подхода NEW проведен по сравнению с дисциплиной обслуживания PRIORL. На рисунке 4а показан средний эффект C алгоритмов выполнения заявок работы без реализации системы адаптивного управления (PRIORL) и с системой адаптивного управления (NEW). Как видно из рисунка 4a, NEW показывает лучший средний эффект C, чем PRIORL. На рисунке 4b показано отношение среднего эффекта NEW к PRIORL и выражено через коэффициент $\eta$. B среднем значение коэффициента $\eta$ составило 2,156 раза по сравнению с альтернативной схемой без управления источниками ресурсов.

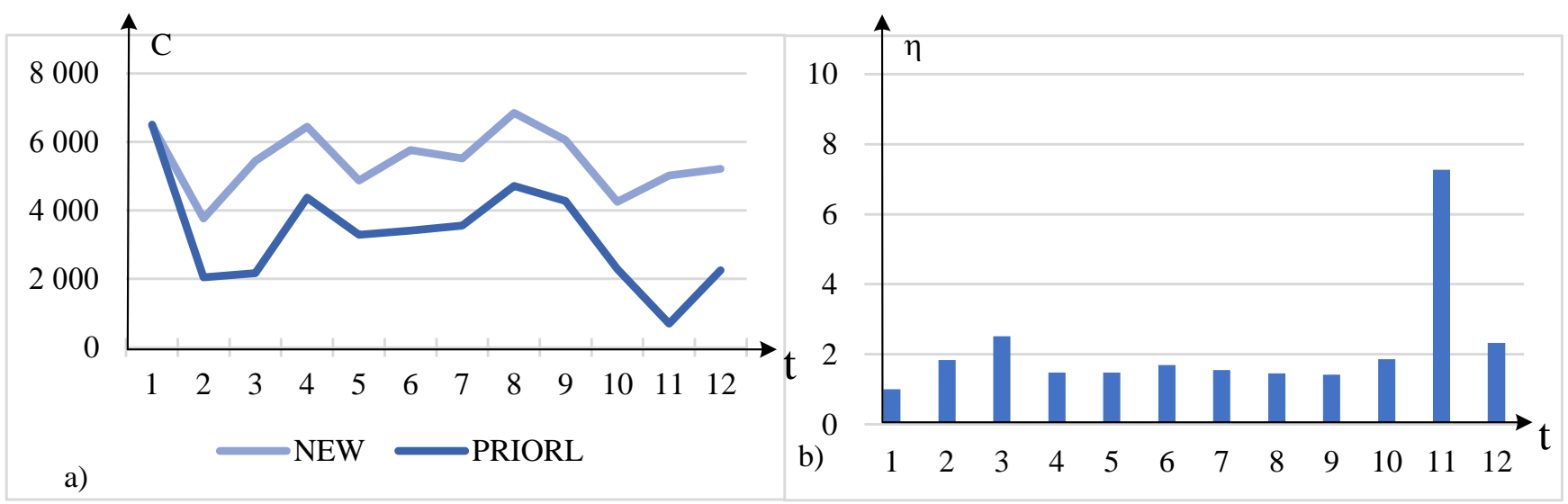

Рис. 4. Средний эффект: а - средний суммарный эффект (на основе функции затрат);

б - коэффициент среднего эффекта

Fig. 4. Average effect: a - average total effect (based on the cost function); $b$ - the coefficient of the average effect

На рисунке 5а для NEW показана зависимость прироста общего эффекта относительно максимального коэффициента $\eta$ для требуемых ресурсов от доступных. На рисунке $5 б$ для NEW показана зависимость общего эффекта С относительно максимального коэффициента $\eta$ для требуемых ресурсов от доступных.

Проведя эксперименты, можно утверждать, что разработанная система адаптивного управления превосходит другие системы. Основной эффект был оценен в рамках алгоритма $<$ PRIORL $>$. Как видно из рисунка 4б, алгоритм $<$ NEW $>$ превосходит алгоритм $<$ PRIORL $>$ с точки зрения совокупного эффекта (суммарной величины стоимостной функции). 


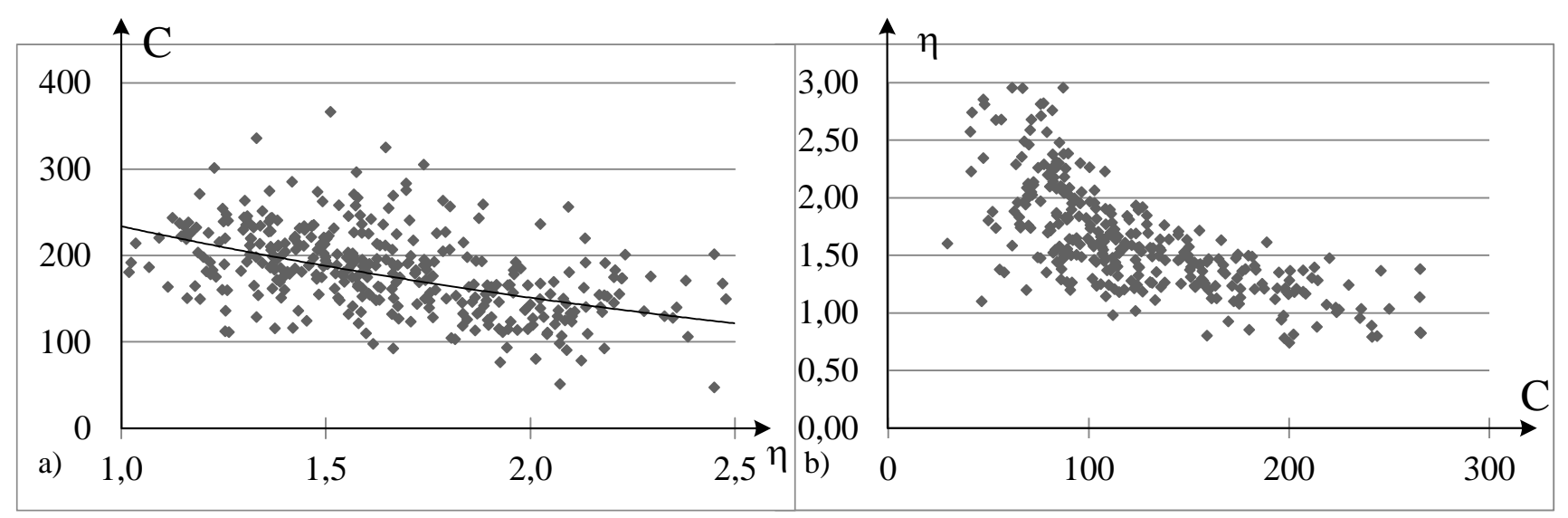

Рис. 5. Общий эффект: а - общий эффект NEW в зависимости от соотношения ресурсов; б - общий коэффициент эффекта в зависимости от предыдущего эффекта

Fig. 5. The overall effect: a - the overall effect of NEW depending on the ratio of resources; $\mathrm{b}$ - the overall effect coefficient depending on the previous effect

\section{Заключение}

В работе предложен вариант адаптивной схемы совместного управления обслуживанием заявок на создание ИТ-сервисов и потоками ресурсов в форме системы с двумя контурами обратной связи. В основном устройстве осуществляется выбор заявок на ИТ-сервисы для их реализации путем максимизации целевой функции. В первом контуре адаптируются правила управления очередью заявок на основе соотношения объемов имеющихся и необходимых ресурсов. Во втором контуре управления формируются заявки на предоставление ресурсов адаптивно к потоку требуемых ресурсов. Сравнительный анализ показал прирост эффекта по сравнению с дисциплиной обслуживания PRIORL без управления ресурсами до 2 раз. Вариант применения данной схемы адаптивного управления был реализован в форме патента [Абдалов, Гришаков, Логинов, 2020] и зарегистрирован, также была зарегистрирована программа для ЭВМ [Абдалов, Гришаков, Логинов, 2019]. Применение описанной системы позволяет повысить эффективность выполнения заявок на создание и модернизацию ИТ-сервисов.

\section{Список литературы}

1. Абдалов А.В., Гришаков В.Г., Логинов И.В. 2020. Патент на изобретение №2729228 Российская Федерация. Способ управления распределением ресурсов в распределенных информационно-вычислительных средах. Заявитель и патентообладатель Федеральное государственное казенное образовательное учреждение высшего профессионального образования «Академия Федеральной службы охраны Российской Федерации». №2019143703 М.: Роспатент.

2. Абдалов А.В, Гришаков В.Г., Логинов И.В. 2019. Программа по расчету оптимальных планов распределения информационно-телекоммуникационных ресурсов организации, динамически поступающих из нескольких источников, между проектами реализации ИТ-сервисов. RU 2019614801.

3. Беллман Р., Дрейфус С. 1965. Прикладные задачи динамического программирования. М.: Наука, 458 с.

4. Бон Я., Кеммерлинг Г., Пондаман Д. 2003. ИТ Сервис-менеджмент, введение. Под ред. М.Ю. Потоцкого (русская версия). М: IT Expert, 215 с.

5. Бурков В.Н., Новиков Д.А. 2004. Как управлять организациями. М.: Синтег, 400 с.

6. Воронин А.А., Губко М.В., Мишин С.П., Новиков Д.А. 2008. Математические модели организаций: Учебное пособие. М.: ЛЕНАНД, 360 с.

7. Воронин Д.Ю., Скатков И.А., Кобылянская М.С. 2014. Модели распределения ресурсов в сервис-ориентированных системах и инфраструктурах. Вестник СевНТУ. 154: 45-48.

8. Горбанева О.И. 2010. Игровые модели распределения ресурсов в иерархических системах управления качеством речной воды. Математическая теория игр и ее приложения. 2(1): 27-46. 
9. Грехов В.А., Ивенин И.Б., Кудрявцев Н.С. 2008. Статическое и динамическое распределение ресурсов. Вестник МГТУ гражданской авиации. 132: 73-80.

10. Карымов В.Р., Славская М.В. 2001. Многомерная линейная модель распределения ресурсов. Математическое образование на Алтае: Труды региональной научно-методической конференции. Барнаул: Изд-во АлГТУ. 33-36.

11. Матвеев А.А. 2005. Модели и методы распределения ресурса при управлении портфелями проектов. Управление большими системами: сборник трудов. 10: 98-106.

12. Могилевский В.Д. 2005. Формализация динамических систем. М., 215 с.

13. Прилуцкий М.X., Власов С.Е. 2005. Многостадийные задачи теории расписаний с альтернативными вариантами выполнения работ. Системы управления и информационные технологии. 2: $44-48$.

14. Lane J.A. 2009. Cost Model Extensions to Support Systems Engineering Cost Estimation for Complex Systems and Systems of Systems. 7th Annual Conf. on Systems Engineering Research, Loughborough University. DOI= http://cser.lboro.ac.uk/papers/S02-14.pdf. (дата обращения 15.10.2012).

15. Weber Ch.A., Current J.R., Benton W.C. 1991. Vendor Selection Criteria and Methods. European Journal of Operational Research.50(1): 2-18.

16. Wei C-C., Chien C-F., Wang M-J. J. 2005. An AHP-based approach to ERP system selection. Int. J. Production Economics. 96: 47-62.

17. Wynne M.W., Schaeffer M.D. 2005. Revitalization of Systems Engineering in DoD. Defense AT\&L. 14-17.

18. Ernstoff, M. and Vincenzini, I. 1999. Guide to Products of System Engineering. International Council on Systems Engineering, Las Vegas.

\section{References}

1. Abdalov A.V., Grishakov V.G., Loginov I.V. 2020. Patent for invention No. 2729228 Russian Federation. A method for managing the distribution of resources in distributed information and computing environments. applicant and patent holder Federal State State Educational Institution of Higher Professional Education "Academy of the Federal Security Service of the Russian Federation". No. 2019143703. M.: Rospatent,

2. Abdalov A.V., Grishakov V.G., Loginov I.V. 2019. Program for calculating optimal plans for the distribution of information and telecommunications resources of the organization, dynamically coming from several sources, between projects for the implementation of IT services. RU 2019614801.

3. Bellman R., Dreyfus S. 1965. Applied problems of dynamic programming. Moscow: Nauka, 458 p.

4. Bon Ya., Kemmerling G., Pondaman D. 2003; IT Service Management, introduction. Edited by M.Y. Potocki (Russian version). Moscow: IT Expert, 215 p.

5. Burkov V.N., Novikov D.A. 2004. How to manage organizations. Moscow: Sinteg, 400 p.

6. Voronin A.A., Gubko M.V., Mishin S.P., Novikov D.A. 2008. Mathematical models of organizations. Moscow, $360 \mathrm{p}$.

7. Voronin D.Yu., Skatkov I.A., Kobylyanskaya M.S. 2014. Models of resource allocation in service-oriented systems and infrastructures. Vesnik SevNTU. 154: 45-48.

8. Gorbaneva O.I. 2010. Game models of resource allocation in hierarchical systems of river water quality management. Mathematical game theory and its applications. 2(1): 27-46.

9. Grekhov V.A., Ivenin I.B., Kudryavtsev N.S. 2008. Static and dynamic distribution of resources. Vestnik MSTU civil Aviation. 132: 73-80.

10. Karymov V.R., Slavskaya M.V. 2001. Multidimensional linear model of resource allocation. Mathematical education in the Altai: Proceedings of the regional scientific and methodological conference. Barnaul: AlSTU Publishing House: 33-36.

11. Matveev A.A. 2005. Models and methods of resource allocation in project portfolio management. Management of large systems: a collection of works. 10: 98-106.

12. Mogilevsky V.D. 2005. Formalization of dynamic systems. Moscow, 215 p.

13. Prilutsky M.Kh., Vlasov S.E. 2005. Multi-stage tasks of the theory of schedules with alternative options for performing work. Management systems and information technologies. 2: 44-48.

14. Lane J.A. 2009. Cost Model Extensions to Support Systems Engineering Cost Estimation for Complex Systems and Systems of Systems. 7th Annual Conf. on Systems Engineering Research, Loughborough University. DOI= http://cser.lboro.ac.uk/papers/S02-14.pdf. (data access: 15.10.2012). 
15. Weber Ch.A., Current J.R., Benton W.C. 1991. Vendor Selection Criteria and Methods. European Journal of Operational Research. 50(1): 2-18.

16. Wei C-C., Chien C-F., Wang M-J. J. 2005. An AHP-based approach to ERP system selection. Int. J. Production Economics. 96: 47-62.

17. Wynne M.W., Schaeffer M.D. 2005. Revitalization of Systems Engineering in DoD. Defense AT\&L. 14-17.

18. Ernstoff, M. and Vincenzini, I. 1999. Guide to Products of System Engineering. International Council on Systems Engineering, Las Vegas.

Конфликт интересов: о потенциальном конфликте интересов не сообщалось.

Conflict of interest: no potential conflict of interest related to this article was reported.

\section{ИНФОРМАЦИЯ ОБ АВТОРАХ}

Абдалов Арсентий Владимирович, аспирант, Академия ФСО России, г. Орел, Россия

Гришаков Видим Геннадьевич, кандидат технических наук, научный сотрудник, Академия ФСО России, г. Орел, Россия

Логинов Илья Валентинович, кандидат технических наук, научный сотрудник, Академия ФСО России, г. Орел, Россия

\section{INFORMATION ABOUT THE AUTHORS}

Arsentii V. Abdalov, Post-Graduate Student, The Academy of Federal Guard Service of the Russian Federation, Orel, Russia

Vadim G. Grishakov, Candidate of Technical Sciences, Researcher, The Academy of Federal Guard Service of the Russian Federation, Orel, Russia

Ilya V. Loginov, Candidate of Technical Sciences, Researcher, The Academy of Federal Guard Service of the Russian Federation, Orel, Russia 